\title{
Forward VNS, Reverse VNS, and Multi-VNS Algorithms for Job-Shop Scheduling Problem
}

\author{
Pisut Pongchairerks \\ Industrial Engineering Program, Faculty of Engineering, Thai-Nichi Institute of Technology, Bangkok 10250, Thailand \\ Correspondence should be addressed to Pisut Pongchairerks; pisut@tni.ac.th
}

Received 19 April 2016; Revised 3 July 2016; Accepted 11 August 2016

Academic Editor: Farouk Yalaoui

Copyright (C) 2016 Pisut Pongchairerks. This is an open access article distributed under the Creative Commons Attribution License, which permits unrestricted use, distribution, and reproduction in any medium, provided the original work is properly cited.

\begin{abstract}
This paper proposes a number of forward VNS and reverse VNS algorithms for job-shop scheduling problem. The forward VNS algorithms are the variable neighborhood search algorithms applied to the original problem (i.e., the problem instance with the original precedence constraints). The reverse VNS algorithms are the variable neighborhood search algorithms applied to the reversed problem (i.e., the problem instance with the reversed precedence constraints). This paper also proposes a multi-VNS algorithm which assigns an identical initial solution-representing permutation to the selected VNS algorithms, runs these VNS algorithms, and then uses the best solution among the final solutions of all selected VNS algorithms as its final result. The aim of the multi-VNS algorithm is to utilize each single initial solution-representing permutation most efficiently and thus receive its best result in return.
\end{abstract}

\section{Introduction}

Job-shop scheduling problem (JSP) is a hard-to-solve scheduling problem which has commonly been found in many industries. JSP is similar to other scheduling problems in the terms that it consists of a number of jobs and a number of machines, and it requires assigning the given jobs into the given machines over time. However, JSP has some more specific constraints which make it unique and thus different from the other scheduling problems. These specific constraints are given as follows. Each job in JSP consists of a number of operations which must be processed in the specific order as the precedence constraints. Each operation must be processed on a preassigned machine by a specific processing time without preemption. In addition, each machine cannot process more than one operation simultaneously. The objective of JSP is to find a feasible schedule which completes all given jobs by the shortest makespan, that is, the time length from the starting time to the completion time of the schedule.

In order to solve JSP, this paper is interested in applying variable neighborhood search (VNS) algorithm because this algorithm is recognized as a simple, systematic, and successful metaheuristic for combinatorial problems. This paper receives insight and motivation from the previously published literature to develop the forward VNS, reverse VNS, and multi-VNS algorithms. The forward VNS algorithms are the VNS algorithms applied to the original problem (i.e., the being-considered JSP instance with the original precedence constraints). The reverse VNS algorithms are the VNS algorithms applied to the reversed problem (i.e., the being-considered JSP instance with the reversed precedence constraints); each reverse VNS algorithm has an additional step to transform its reversed problem's solution to be usable for the original problem. The proposed multi-VNS algorithm is an algorithm which assigns the same initial solutionrepresenting permutation into a number of the specified VNS algorithms, runs these VNS algorithms, and finally uses the best solution among the final solutions of these VNS algorithms as its final result. In other words, the multiVNS algorithm aims at utilizing each single initial solutionrepresenting permutation most efficiently by systematically using different VNS neighborhood structures and scheduling directions on the same initial solution-representing permutation.

The remaining parts of this paper are organized as follows. Section 2 reviews the articles related to the research in this paper and then summarizes the research contributions. Section 3 proposes a generic VNS algorithm, so that this 
section later proposes the forward VNS algorithms and the reverse VNS algorithms based on the given generic form. This paper proposes a multi-VNS algorithm in Section 4 and then evaluates the multi-VNS algorithm's performance in Section 5. Section 6 finally provides the conclusions of this research.

\section{Literature Review and Research Contribution}

Job-shop scheduling problem (JSP) starts with $n$ given jobs $J_{1}, J_{2}, \ldots, J_{n}$ and $m$ given machines $M_{1}, M_{2}, \ldots, M_{m}$. Each job $J_{i}$ is composed of $m$ given operations $O_{i 1}, O_{i 2}, \ldots, O_{i m}$ which must be processed in the given order as a chain of precedence constraints. This means, for each job $J_{i}$, the operation $O_{i 1}$ must be finished before the operation $O_{i 2}$ can be started, the operation $\mathrm{O}_{i 2}$ must be finished before the operation $\mathrm{O}_{i 3}$ can be started, and so on. The operation $O_{i j}$ can be processed only on a preassigned machine with a specific preassigned processing time. The preemption for each operation is not allowed; that is, after a particular machine starts processing any operation, it cannot be stopped or paused for any reasons until finishing the operation. In addition, each machine can process only one operation at a time. JSP aims at finding a schedule (i.e., an allocation of all given operations to time intervals on the given machines) which satisfies all abovegiven constraints so as to minimize the schedule's makespan. As mentioned, the makespan defines the time length from the starting time of the schedule (i.e., the starting time of the first started operation in the schedule) to the completion time of the schedule (i.e., the completion time of the last finished operation in the schedule). JSP is a well-known scheduling problem, so it has been mentioned frequently in the textbooks, for example, $[1,2]$. The mathematical models for describing JSP have been commonly found in literature, for example, [3].

JSP is important to industry and attractive to academia, so many algorithms have been developed for solving the problem. These algorithms include tabu search algorithms $[4,5]$, a simulated annealing algorithm (SA) [6], a hybrid algorithm between particle swarm optimization (PSO) and VNS [7], genetic algorithms (GAs) [8-13], PSO algorithms [14-16], VNS algorithms [17-19], a hybrid algorithm between PSO and GA [20], a bee colony algorithm [21], an ant colony optimization algorithm [22], a memetic algorithm [23], and a hybrid algorithm between GA and SA [24]. Based on the literature review, the VNS algorithms are recognized as wellperforming algorithms for JSP, so this paper will research more on the VNS algorithms.

VNS was first introduced in $[25,26]$ as a metaheuristic approach for combinatorial optimization problems. As its name implies, VNS changes its neighborhood structure from one to another systematically in the purpose of finding local optimal solutions as well as escaping from them, so VNS is highly potential to find a global optimal solution. The development of VNS is based on the three following observations $[25,27]$ :
(1) A local optimal solution in a neighborhood structure may not be the same as a local optimal solution in another neighborhood structure.

(2) A global optimal solution is a local optimal solution with respect to all possible neighborhood structures.

(3) In many problem instances, local optimal solutions in different neighborhood structures are relatively close to each other.

VNS generally consists of three main steps: the shaking step, the local search step, and the step of updating its best found solution. The VNS algorithm's local search step aims at finding a local optimal solution with respect to variable neighborhood structures. The shaking step aims at escaping from a local optimal solution as well as generating a new initial solution for the local search step. In published literature, the review articles about VNS are found in [25-28], the applications of VNS are found in [17-19, 28, 29], and the parallelization strategies for VNS are given in [27, 30].

The articles closely related to the research in this paper are articles [7, 17, 19, 29]. As mentioned above, a wellperforming hybrid algorithm between PSO and VNS for JSP was given in [7]. Later, article [17] disassembled the VNS from the hybrid algorithm and reported that the VNS algorithm alone performs as equally well as the hybrid algorithm in terms of solution quality. After that, article [29] introduced the variants of the VNS algorithm of [17] for asymmetric traveling salesman problem, while article [19] introduced the variants of the VNS algorithm of [17] for JSP. Article [19] is most closely related to the research in this paper, because this paper aims at enhancing the performances of the VNS algorithms given in [19].

As just mentioned, the objective of the research in this paper is to enhance the performances of the VNS algorithms of [19], so the contributions of the research in this paper are given in overview as follows. A preliminary study of this research finds that, in several hard-to-solve JSP instances, the maximum iterations of the VNS algorithms of [19] should be increased in order to enhance their potentials of finding the optimal solutions; thus, this paper will find out the more proper maximum iterations for the VNS algorithms. This paper will also introduce more variants of the VNS algorithms of [19] which use different neighborhood structures from [19]. Moreover, this paper will introduce the use of the reversed problem (i.e., the being-considered JSP instance with the reversed precedence constraints) for the VNS algorithms because each hard-to-solve JSP instance may be solved easier in its corresponding reversed problem. Note that the schedule's construction using the reversed precedence constraints is called the reverse or backward scheduling, and it has often been applied for scheduling problems in many articles such as $[14,22,31,32]$. For efficiently utilizing each initial solution-representing permutation, this paper will then propose the multi-VNS algorithm which assigns an identical initial solution-representing permutation into the selected VNS algorithms, runs these VNS algorithms, and uses the best solution found by these VNS algorithms as its final solution. 


\section{Proposed VNS Algorithms}

This section will propose the generic VNS algorithm for JSP, so that the forward VNS algorithms and the reverse VNS algorithms will be developed based on the given generic VNS algorithm. As mentioned earlier, the forward VNS algorithms define the VNS algorithms applied to the original problem, that is, the being-considered JSP instance using the original (forward) precedence constraints. The reverse VNS algorithms define the VNS algorithms applied to the reversed problem, that is, the being-considered JSP instance using the reverse (backward) precedence constraints. In addition, each reverse VNS algorithm has one more additional step to modify its reversed problem's solution to be usable for the original problem. The terms forward VNS and reverse VNS will hereafter be abbreviated by FVNS and RVNS, respectively. Section 3.1 provides the generic VNS algorithm which is the generic form for all FVNS and RVNS algorithms proposed in this paper. Based on the generic form given, Section 3.2 presents the FVNS and RVNS algorithms using different operators to generate their solution-representing permutations. The performances of the proposed FVNS and RVNS algorithms will also be tested in Section 3.2.

3.1. Generic VNS Algorithm for JSP. This section introduces the generic VNS algorithm as the generic form of the FVNS and RVNS algorithms proposed later in Section 3.2. Note that the solutions (i.e., the JSP schedules) generated by all proposed VNS algorithms are represented by the operation-based permutations [3, 13]. Each operation-based permutation is an arrangement of the $m n$ integers consisting of $1_{1}, 1_{2}, \ldots, 1_{m}, 2_{1}, 2_{2}, \ldots, 2_{m}, \ldots, n_{1}, n_{2}, \ldots, n_{m}$, where the subscripts are used to distinguish the integer of the same value. In other words, it is a sequence of the $m n$ integers consisting of the numbers from 1 to $n$, where each number (from 1 to $n$ ) occurs repeatedly $m$ times. Based on the JSP definition given in Section 2, $n$ is the number of all given jobs, while $m$ is the number of all given machines. Moreover, $m$ is also equal to the number of all operations of each job, so $m n$ is thus the number of all operations in the schedule. As an example, $P=\{2,3,2,1,1,3\}$ is an operation-based permutation possibly generated by a particular VNS algorithm for a 3-job/2-machine JSP instance. The procedure to decode an operation-based permutation into a JSP schedule, as found in $[3,13,17,19]$, is given here in Algorithm 1. This decoding procedure transforms the operation-based permutation into an order of priorities of all given operations and then uses this order of priorities to construct a semiactive schedule. Note that a semiactive schedule is a feasible schedule such that no operations can be started earlier without altering the given order of priorities of operations.

Algorithm 1. It is a procedure to decode an operation-based permutation into a semiactive schedule.

Step 1. Let $P$ represent the operation-based permutation which is required to be transformed into the semiactive schedule $S$. Let the number $i(i=1,2, \ldots, n)$ in the $j$ th occurrence $(j=1,2, \ldots, m)$ from leftmost to the right of the permutation $P$ refer to the $j$ th operation of the job $i$ or $O_{i j}$. After that, let the order of these $m n$ operations in the permutation $P$ from leftmost to the right define the order of priorities of the $m n$ operations from highest to lowest. For example, $P=\{2,3,2,1,1,3\}$ means that the order of priorities of the operations in descending order is $O_{21}, O_{31}, O_{22}, O_{11}$, $\mathrm{O}_{12}$, and $\mathrm{O}_{32}$.

Step 2. At the beginning, let the schedule $S$ be empty, so the earliest available times of all $m$ machines equal 0 . Let $t=0$.

Step 3. Based on the order of priorities of all operations given in Step 1, let $O$ represent the current highest-priority operation among all as-yet-unassigned operations. Then, let $\tau$ represent the preassigned processing time of the operation $O$, and let $M$ represent the preassigned machine required by the operation $O$.

Step 4. Assign the operation $O$ into the schedule $S$ by letting the starting time of the operation $O$ equal the maximum between the earliest available time of the machine $M$ and the completion time of the immediate-predecessor operation of the operation $O$. As a consequence, the completion time of the operation $O$ equals its starting time given in this step plus its processing time $\tau$.

Step 5. Update the earliest available time of the machine $M$ to equal the completion time of the operation $O$.

Step 6. Increase $t$ by 1 . After that, if $t=m n$, stop and the schedule $S$ is now completely constructed with the makespan equal to the maximum of the completion times of all $m n$ operations; otherwise, repeat from Step 3.

Algorithm 2 is the procedure of the generic VNS algorithm which uses Algorithm 1 to decode operation-based permutations into JSP solutions. $P_{0}, P_{1}$, and $P_{2}$ in Algorithm 2 are the operation-based permutations which represent the job-shop schedules $S_{0}, S_{1}$, and $S_{2}$, respectively. The permutation $P_{0}$ is the current best found permutation, so the schedule $S_{0}$ is the current best found solution. As mentioned, each permutation of $P_{0}, P_{1}$, and $P_{2}$ is a sequence of $m n$ integers consisting of the numbers $1,2,3, \ldots, n$, where each number (from 1 to $n$ ) is repeatedly $m$ times. The solution neighborhood structures used by the generic VNS algorithm are generated based on the swap operator and the insert operator. The swap (i.e., interchange) and insert (i.e., shift) operators are commonly used in literature, for example, $[7,21$, 33]. In this paper, the swap operator generates a neighbor of a specific operation-based permutation by randomly selecting two integers (of all $m n$ integers) from two different positions in the permutation and then swapping the positions of the two selected integers. The insert operator is done by randomly selecting two integers (of all $m n$ integers) from two different positions in the permutation, removing the first-selected integer from its old position, and then inserting it into the position in front of the second-selected integer. 
Algorithm 2. It is a procedure of generic VNS algorithm.

Step 1. Let the user specify the type of this VNS algorithm to be a forward VNS algorithm or a reverse VNS algorithm and specify each of the operators $A, B, C$, and $D$ to be the swap operator or the insert operator. If this VNS algorithm is specified to be a reverse VNS algorithm, then generate the reversed problem by letting the machine and the processing time of its operation $O_{i j}(i=1,2, \ldots, n$ and $j=1,2, \ldots, m)$ be equal to the machine and the processing time of the operation $O_{i k}(i=1,2, \ldots, n$ and $k=m-j+1)$ of the original problem; then, replace the original problem by the reversed problem in all the following steps.

Step 2. Generate $P_{0}$ randomly as an initial current best operation-based permutation (or, as an option, $P_{0}$ can also be generated manually by the user) and then transform $P_{0}$ into the job-shop schedule $S_{0}$ by using Algorithm 1. Let the VNS algorithm's iteration $t=0$.

Step 3. Process the shaking step by generating $P_{1}=$ $A\left(A\left(B\left(B\left(P_{0}\right)\right)\right)\right)$ and then transforming $P_{1}$ into a job-shop schedule $S_{1}$ by using Algorithm 1.

Step 4. Let the local search procedure's iteration $t_{\text {local }}=0$. Process the local search procedure by Steps 4.1 to 4.5 .

Step 4.1. Let $l_{\max }=2$ and $l_{\text {count }}=0$.

Step 4.2. If $l_{\text {count }}=0$, then generate $P_{2}=C\left(P_{1}\right)$; however, if $l_{\text {count }}=1$, then generate $P_{2}=D\left(P_{1}\right)$ instead. After that, transform $P_{2}$ into the job-shop schedule $S_{2}$ by using Algorithm 1.

Step 4.3. If the makespan of $S_{2}>$ the makespan of $S_{1}$, then increase $l_{\text {count }}$ by 1 . However, if the makespan of $S_{2} \leq$ the makespan of $S_{1}$, then update $l_{\text {count }}$ to equal 0 , update $P_{1}$ to equal $P_{2}$, and also update $S_{1}$ to equal $S_{2}$.

Step 4.4. If $l_{\text {count }}=l_{\text {max }}$, then go to Step 4.5; otherwise, repeat from Step 4.2.

Step 4.5. Increase $t_{\text {local }}$ by 1 . After that, if $t_{\text {local }}=m n(m n-1)$, then go to Step 5; otherwise, repeat from Step 4.1.

Step 5. If the makespan of $S_{1} \leq$ the makespan of $S_{0}$, then update $P_{0}$ to equal $P_{1}$ and also update $S_{0}$ to equal $S_{1}$.

Step 6. Increase $t$ by 1 . After that, check the conditions below:

(i) If the stopping criterion is not met, then repeat from Step 3.

(ii) If the stopping criterion is met and this VNS algorithm is specified in Step 1 to be a forward VNS algorithm, then stop and $S_{0}$ is the final solution.

(iii) If the stopping criterion is met and this VNS algorithm is specified in Step 1 to be a reverse VNS algorithm, then go to Step 7 .
Step 7. Transform $S_{0}$ into $S_{R 0}$ by letting the starting time of $O_{i j}$ $(i=1,2, \ldots, n$ and $j=1,2, \ldots, m)$ of $S_{R 0}$ equal the makespan of $S_{0}$ minus the completion time of $O_{i k}(i=1,2, \ldots, n$ and $k=m-j+1)$ of $S_{0}$. As a consequence, the completion time of $O_{i j}(i=1,2, \ldots, n$ and $j=1,2, \ldots, m)$ of $S_{R 0}$ then equals the makespan of $S_{0}$ minus the starting time of $O_{i k}(i=1,2, \ldots, n$ and $k=m-j+1)$ of $S_{0}$. Then, stop and $S_{R 0}$ is the final solution. Note that the makespan of $S_{R 0}$ is equal to the makespan of $S_{0}$.

The main steps of the generic VNS algorithm in Algorithm 2 are more clarified as follows. Step 1 requires the user to assign the type of the VNS algorithm which can be either a forward VNS algorithm or a reverse VNS algorithm. If the type of VNS algorithm is specified to be a reverse VNS algorithm, the precedence constraints of all operations of each job must be reversed. In Step 1, the user moreover has to specify that each of the operators $A, B, C$, and $D$ is either the swap operator or the insert operator. Step 2 in Algorithm 2 generates $P_{0}$ as the initial current best operationbased permutation and then transforms the permutation $P_{0}$ into the schedule $S_{0}$ through the decoding procedure given in Algorithm 1.

Step 3 is the shaking step of the VNS algorithm, which modifies the operation-based permutation $P_{0}$ to the operation-based permutation $P_{1}$. To do so, Step 3 uses the operator $B$ to $P_{0}$ for receiving the permutation $B\left(P_{0}\right)$, then uses the operator $B$ again to the permutation $B\left(P_{0}\right)$ for receiving the permutation $B\left(B\left(P_{0}\right)\right)$, then uses the operator $A$ to the permutation $B\left(B\left(P_{0}\right)\right)$ for receiving the permutation $A\left(B\left(B\left(P_{0}\right)\right)\right)$, and finally uses the operator $A$ again to the permutation $A\left(B\left(B\left(P_{0}\right)\right)\right)$ for receiving the permutation $A\left(A\left(B\left(B\left(P_{0}\right)\right)\right)\right)$ as $P_{1}$.

Step 4 in Algorithm 2 is the VNS algorithm's local search procedure used to improve the permutation $P_{1}$; thus, at the end of Step 4, $P_{1}$ will be a local optimal solution. Later, Step 5 checks whether the permutation $P_{1}$ taken from Step 4 is better than or equal to the current best permutation $P_{0}$ or not. If so, the current best permutation $P_{0}$ will be updated to equal the permutation $P_{1}$.

Step 6 is the step to check whether the stopping criterion is satisfied or not and check whether this VNS algorithm is a forward VNS algorithm or a reverse VNS algorithm. If the stopping criterion is not satisfied, then the VNS algorithm will process its next iteration. If the stopping criterion is satisfied and this VNS algorithm is a forward VNS algorithm, then stop and the schedule $S_{0}$ is the final solution. If the stopping criterion is satisfied and this VNS algorithm is a reverse VNS algorithm, then process Step 7 in order to transform the schedule $S_{0}$ into the schedule $S_{R 0}$, which is the reverse VNS algorithm's final solution usable for the original problem.

3.2. Proposed Forward VNS and Reverse VNS Algorithms. As previously mentioned, Algorithm 2 is the generic form for all forward VNS (FVNS) algorithms and all reverse VNS (RVNS) algorithms proposed in this section. Each of $A, B, C$, and $D$ in Algorithm 2 can be specified to be either the swap operator or the insert operator, so there are a total of $32 \mathrm{VNS}$ algorithms generated from Algorithm 2 including 16 FVNS 
algorithms and 16 RVNS algorithms. For the identification purpose, let us name these $32 \mathrm{VNS}$ algorithms in the format of TABCD. Let $T$ represent the specified VNS type which can be either $F=$ a forward VNS algorithm or $R=$ a reverse VNS algorithm. Let $A, B, C$, and $D$ here represent the specified operators for the generic operators $A, B, C$, and $D$ in Algorithm 2, so each of $A, B, C$, and $D$ can be either $S$ $=$ the swap operator or $I=$ the insert operator. For example, FISIS refers to the forward VNS algorithm in which $A$ is the insert operator, $B$ is the swap operator, $C$ is the insert operator, and $D$ is the swap operator; RSISS means the reverse VNS algorithm in which $A$ is the swap operator, $B$ is the insert operator, $C$ is the swap operator, and $D$ is the swap operator.

Based on the format of $T A B C D$ above given, the 16 FVNS algorithms include FSSSS, FSSSI, FSSIS, FSSII, FSISS, FSISI, FSIIS, FSIII, FISSS, FISSI, FISIS, FISII, FIISS, FIISI, FIIIS, and FIIII, and the 16 RVNS algorithms include RSSSS, RSSSI, RSSIS, RSSII, RSISS, RSISI, RSIIS, RSIII, RISSS, RISSI, RISIS, RISII, RIISS, RIISI, RIIIS, and RIIII. Note that the eight FVNS algorithms, that is, FIIIS, FIISI, FISIS, FISSI, FSIIS, FSISI, FSSIS, and FSSSI, are slightly modified from the VNS algorithms of [19] in that their maximum iterations are extended from the 250th iteration to the 1,000th iteration. The modification just mentioned is due to the result of this paper's preliminary study which finds that the maximum iteration of 250th iteration makes each VNS algorithm stop prematurely before receiving its best returns in several hard-tosolve instances. The discussion about the proper maximum iteration will be given at the end of this section. In addition, FSISI is also found in [17] with a slightly different stopping criterion from the criterion used here; FSISI can thus be recognized as the original variant of all VNS algorithms given in this paper.

The 16 FVNS and 16 RVNS algorithms proposed in this section are compared in their performances on the 43 wellknown benchmark instances, that is, $\mathrm{ft} 06, \mathrm{ft} 10$, and $\mathrm{ft} 20$ from [34] and la01-la40 from [35]. The number of all jobs $(n)$ and the number of all machines $(m)$ of all instances are given in parentheses in the form of instance's name $(n, m)$ as follows: ft06 $(6,6), \mathrm{ft} 10(10,10)$, ft20 $(20,5)$, la01-la05 $(10,5)$, la06-la10 (15, 5), la11-la15 $(20,5)$, la16-la20 $(10,10)$, la21-la25 $(15,10)$, la26-la30 $(20,10)$, la31-la35 $(30,10)$, and la36-la40 $(15,15)$. In the experiment here, the proposed FVNS and RVNS algorithms are all coded in C\# and executed on an Intel(R) Core(TM) i5 CPU processor M580 2.67 GHz. These VNS algorithms will be stopped when either the 1,000th iteration as the maximum iteration is reached or the optimal solution given by the published literature $[7,8,24]$ is found. In other words, the stopping criterion in Step 6 of each VNS algorithm is either the VNS algorithm's iteration $t=1,000$ or the makespan of $S_{0}=$ the optimal solution value given by the published literature. All VNS algorithms will be run once on each given instance with the same random seed number and the same initial operation-based permutation. For each instance, this paper uses the solution value deviation to evaluate the quality of the final solution given by each algorithm. For a specific instance, the solution value deviation is equal to $100 \% \times$ (the algorithm's final solution value - the optimal solution value)/the optimal solution value. Thus, if the algorithm can reach the optimal solution value given in the published literature, the solution value deviation is then $0.000 \%$. Note that, in this paper, a solution refers to a schedule and a solution value refers to a schedule's makespan.

Table 1 shows the solution value deviation (\%) of every proposed VNS algorithm over a single run for each instance. The row Best, the last row in Table 1, provides the best solution value deviation found by all proposed VNS algorithms on each instance. The column Avg, the last column in Table 1, provides the average solution value deviation of all 43 instances of each VNS algorithm. Note that the instance will be absented from Table 1 if all 32 VNS algorithms can return the solution value deviations of $0.000 \%$ for it. This means Table 1 reports that all 32 VNS algorithms can reach the optimal solutions for the 28 instances of the total 43 instances, that is, ft06, la01-la15, la17-la19, la23, la26, la28, and la30la35. Based on the results in Table 1, the list of the 32 VNS algorithms in ascending order of their average solution value deviations is FSSII, FISIS, FSSSI, RSSIS, RSISI, RISSI, FIIII, RIISI, RSSSI, FSIII, RIISS, FISSS, RSSSS, RISIS, RISII, RIIII, FSIIS, RIIIS, FIIIS, RSSII, RSIII, FIISI, FSSIS, RSIIS, FSSSS, FSISI, FISSI, RISSS, FIISS, FISII, RSISS, and FSISS.

The comparison results in terms of speed given in Table 2 indicate that the computational times per iteration of all proposed VNS algorithms are not significantly different. However, the total computational times of each VNS algorithm may differ from one another because the VNS algorithm will stop before reaching the maximum iteration if it can find the optimal solution. (Remember that the stopping criterion is to stop if either the optimal solution given by the published literature is found or the maximum iteration is reached.) Thus, the VNS algorithm performing better in solution quality tends to perform better in speed as well. However, if the stopping criterion is changed to be considered only on the maximum iteration, the speeds of all proposed VNS algorithms will differ very slightly. Table 2 shows the computational times and the number of iterations used by FSSII, FISIS, FSSSI, RSSIS, and RSISI which are the five bestperforming VNS algorithms in Table 1. In Table 2, Number of iters means the number of all iterations used until the stopping criterion is met, CPU time (sec.) means the total computational time used until the stopping criterion is met, and the CPU time/iter means the computational time per iteration.

Figure 1 then reveals the proper maximum iterations for the proposed VNS algorithms. Based on the same data source used in Table 1, Figure 1 provides the average-solutionvalue-deviation-over-iteration plots of FSSII, FISIS, FSSSI, RSSIS, and RSISI on all 43 instances. Their average-solutionvalue-deviation-over-iteration plots are all formed in similar patterns which are reduced rapidly before the 500th iteration and then reduced slowly during the 500th iteration to 800th iteration. After that, the average solution value deviations of FSSII, FISIS, FSSSI, and RSSIS have not been improved after the 800th iteration, while RSISI is the only VNS algorithm which can improve its average solution value deviation until the 900th iteration. Based on this observation, this paper thus suggests that the maximum iterations of the proposed VNS algorithms should be in between the 800th iteration and the 
TABLE 1: Solution value deviations (\%) of 16 FVNS and 16 RVNS algorithms.

\begin{tabular}{|c|c|c|c|c|c|c|c|c|c|c|c|c|c|c|c|c|}
\hline \multirow{2}{*}{ Algor } & \multicolumn{15}{|c|}{ Instance } & \multirow{2}{*}{ vg } \\
\hline & 0 & 20 & 16 & 20 & 21 & ב2 & 24 & la25 & la27 & 29 & 6 & 37 & 38 & 39 & 440 & \\
\hline FSSSS & 000 & 00 & 00 & 00 & 96 & & & & & 109 & 173 & 716 & 00 & 00 & \pm 91 & \\
\hline SSSI & 000 & 00 & 00 & 00 & 96 & 00 & 321 & 00 & 43 & 128 & 000 & 716 & 000 & 000 & 164 & 062 \\
\hline FSSIS & 000 & 000 & 000 & 00 & 96 & 24 & 321 & 000 & 243 & 476 & 000 & 002 & 920 & 324 & 164 & 113 \\
\hline FSSII & 00 & 00 & 00 & 00 & & & 21 & 0 & & 42 & 00 & 000 & 920 & 000 & 64 & 057 \\
\hline & & 0 & 00 & 00 & & & & & & 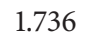 & 00 & & 20 & 00 & & 144 \\
\hline & & 00 & 00 & & & & & & & 55 & 00 & 16 & 18 & 00 & & 119 \\
\hline , & 00 & 000 & 06 & 00 & 69 & & 1 & & & 68 & 00 & 00 & 20 & 00 & & 094 \\
\hline SIII & 00 & 00 & 00 & 00 & & & & & & & & & & & & 083 \\
\hline$C_{1}$ & & & & & & & & & & & & & & & & 086 \\
\hline IC & 60 & 00 & 00 & 00 & 00 & 1 & $2 \pi$ & 12 & 05 & 2.170 & 00 & 00 & 585 & 000 & . & .124 \\
\hline & & & & & & & & & & & & & & & & 060 \\
\hline SII & 00 & 000 & 000 & 54 & & & 21 & 00 & & 36 & 00 & 16 & 20 & 00 & & .131 \\
\hline ISS & 753 & 000 & 000 & 00 & 00 & 00 & 321 & 205 & & 910 & 0.000 & 000 & 000 & 973 & & .127 \\
\hline IISI & 000 & 1.116 & 000 & 54 & 96 & 00 & 321 & 07 & 00 & 955 & 000 & 716 & 418 & 000 & 27 & 112 \\
\hline $\mathrm{s}$ & & 0.000 & 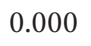 & 0 & & & 0.321 & & & & & & & 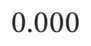 & & 098 \\
\hline ГUU & & & & & & & & & & & & & & & & .077 \\
\hline RSSS & & & & & & & 535 & & & & & & & & & 090 \\
\hline SSS & & & & & & & & & & & 79 & & & & & 080 \\
\hline SSIS & 00 & 00 & 00 & 00 & & & 00 & 0 & 00 & & 0 & & 00 & 00 & & .066 \\
\hline SSII & 00 & 00 & 00 & 00 & 69 & 0 & 000 & 12 & & 3 & 00 & 00 & 20 & 00 & & 0.099 \\
\hline IS & & 00 & 00 & & & & 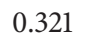 & & & & & & & & & 135 \\
\hline SISI & & 00 & 00 & & & & 1 & & & & & & & & & .073 \\
\hline & & & & & & & & & & & & & & & & 116 \\
\hline RSIII & 0.860 & & & & 1.243 & 0 & 321 & 0.000 & 0.000 & 1.042 & 0.000 & .000 & 920 & 000 & 64 & 106 \\
\hline ISSS & 000 & .000 & 0.000 & 0.000 & 0.096 & 00 & 0.428 & 0.512 & 4 & 1.910 & 00 & 716 & 418 & 00 & 64 & .125 \\
\hline RISSI & 00 & 0.000 & 0.000 & 0.000 & 74 & 0 & 0.3 & 0. & 00 & 1. & 00 & 00 & 18 & 00 & 64 & .074 \\
\hline RISIS & & .000 & & & & & & & & & & & & & & 0.090 \\
\hline RISII & & .000 & 0.000 & 0.000 & & .000 & 0.642 & 0.000 & & & & & & & 0.164 & 0.090 \\
\hline & & 0.000 & 0.000 & 0.000 & & & 0.321 & 0.512 & & & 0.000 & 00 & & 0.000 & 164 & 0.085 \\
\hline RIISI & 0.000 & 0.000 & 0.106 & 54 & 82 & 00 & 0.321 & 0.512 & 0.000 & 1.302 & 0.000 & 0.000 & 0.000 & 0.000 & 0.164 & .078 \\
\hline RIIIS & 0.860 & 0.000 & & & & & 0. & 0.0 & & & 0.000 & 0.000 & 0.920 & 0.000 & 0.164 & 0.097 \\
\hline RII & & 0 & . & 0 & & & & 0.0 & & 1.3 & 0 & 0 & 00 & & & .093 \\
\hline Best & 0.000 & .000 & .000 & 0.000 & 0.000 & .000 & .000 & 0.000 & 0.000 & 0.694 & 0.000 & 0.000 & 0.000 & 0.000 & 0.164 & 0.02 \\
\hline
\end{tabular}

Remark: (i) the solution value deviations of the remaining 28 instances from the total 43 instances, which are not shown in Table 1, are $0.000 \%$ for all 32 VNS algorithms.

(ii) Avg refers to the average solution value deviation over all 43 instances for each VNS algorithm.

1,000 th iteration. In case of requiring the short computational time, the maximum iteration is suggested to be the 500th iteration.

\section{Proposed Multi-VNS Algorithm}

In a brief explanation, the generic multi-VNS algorithm starts by randomly generating an operation-based permutation and then assigning this permutation into the multiple selected VNS algorithms as their initial current best operation-based permutations. Then, the multi-VNS algorithm runs these
VNS algorithms and uses the best solution among the final solutions of these VNS algorithms as its final solution. The development of the multi-VNS algorithm proposed in this paper is motivated by four observations as follows:

(1) On the same JSP instance, two VNS algorithms with different settings of operators $A, B, C$, and $D$ may not perform equally well. This property may also be true even when these two VNS algorithms both use the same initial operation-based permutation as well as the same random seed number. For example, according to Table 1, FSSII can find the optimal 


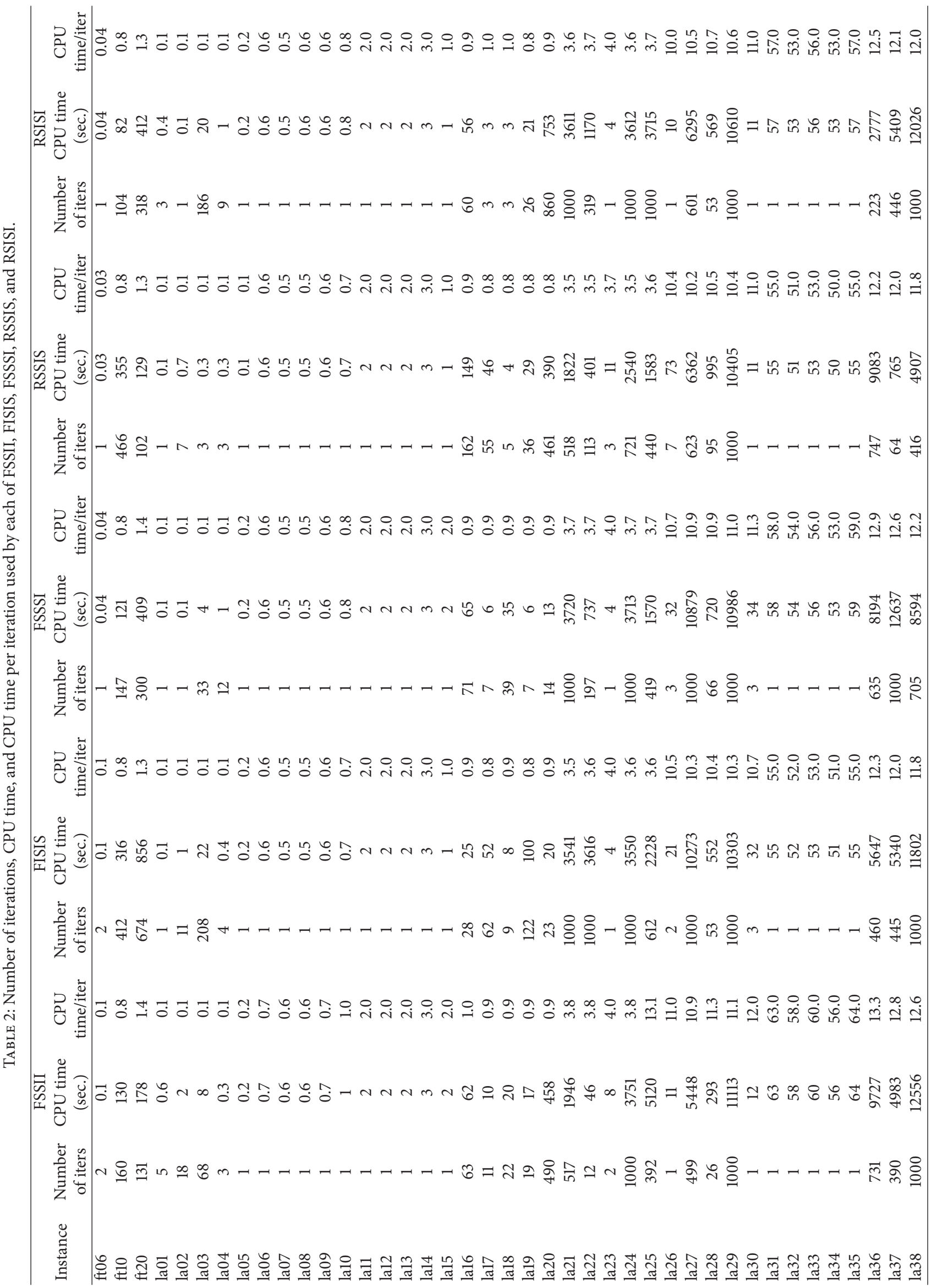




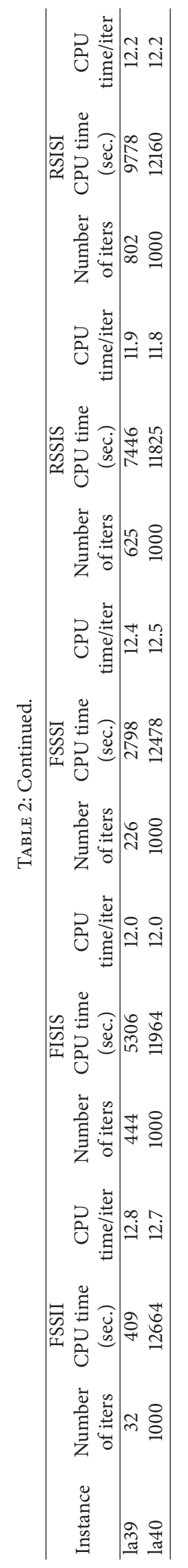




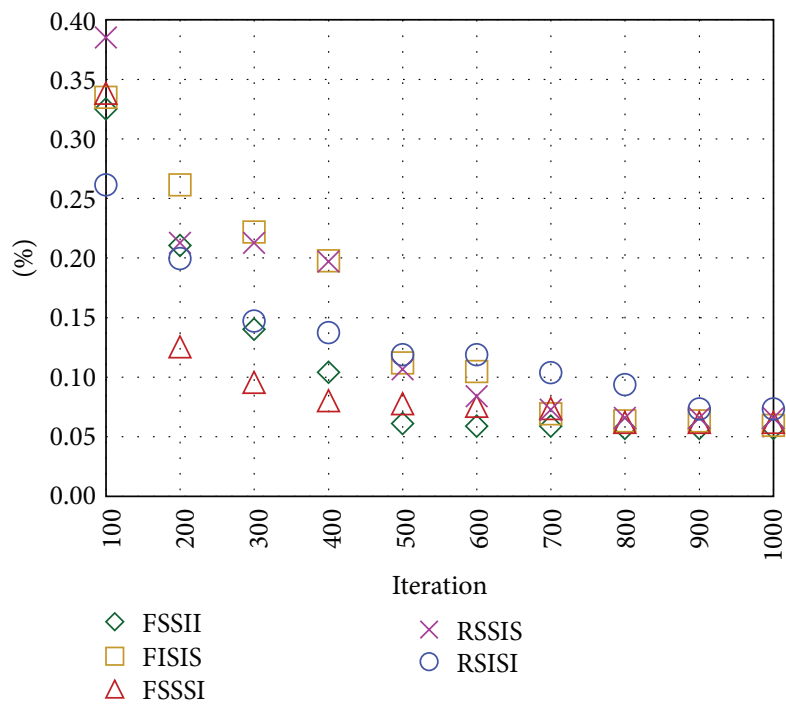

FigURE 1: Average-solution-value-deviation-over-iteration plots of FSSII, FISIS, FSSSI, RSSIS, and RSISI.

solution for la37, while FSSIS cannot; FSSSS can find the optimal solution for la39, while FISSS cannot.

(2) A VNS algorithm which performs well on a particular instance may not perform as well on another instance. For example, FSSII can find the optimal solution for la37, but it cannot find the optimal solution for la29; FSISI can find the optimal solution for $\mathrm{ft} 20$, but it cannot find the optimal solution for $\mathrm{ft} 10$.

(3) A JSP instance which is hard to solve in its original form may be easier to be solved in its reversed problem, and vice versa. For example, according to Table 1, FSSII cannot find the optimal solution for la24, while RSSII can; FIIII can find the optimal solution for $\mathrm{ft} 10$, but RIIII cannot.

(4) It is impossible or very difficult to identify which scheduling direction (forward or reverse) is more efficient for a specific instance without experiments.

The aim of developing the multi-VNS algorithm is to handle with the four above-mentioned observations, and thus the multi-VNS algorithm should utilize each single operation-based permutation most efficiently. The generic multi-VNS algorithm, as shown in Figure 2, starts its process by generating $P_{0}$ as an identical initial operation-based permutation for the $N$ VNS algorithms, that is, the 1st VNS, the 2 nd VNS, ..., the $N$ th VNS. These $N$ VNS algorithms are recommended to be different in their combinations of the $A$, $B, C$, and $D$ operators as well as their VNS types (i.e., forward VNS or reverse VNS). The multi-VNS algorithm then runs each of these specified $N$ VNS algorithms once on the beingconsidered JSP instance. After that, the best solution among the final solutions of all $N$ given VNS algorithms is used as the final result of the multi-VNS algorithm and is abbreviated as $S^{*}$.

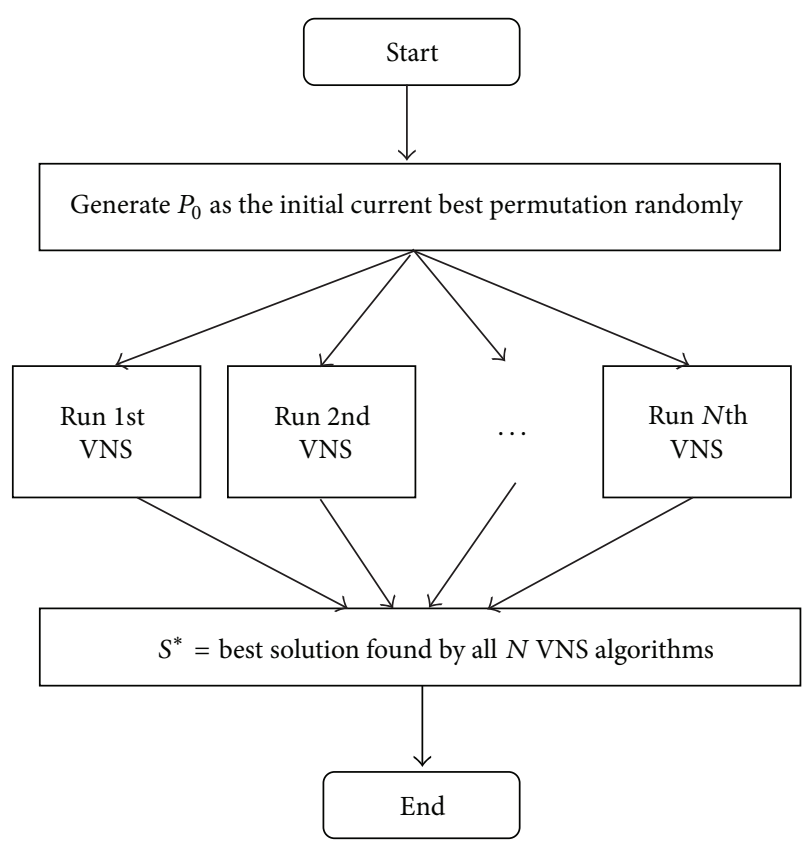

FIGURE 2: Flowchart of the generic multi-VNS algorithm.

Although the proposed multi-VNS algorithm shown in Figure 2 can be run either in sequence or in parallel processing, this paper focuses only on the multi-VNS algorithm operated in sequence via a single stand-alone processor. Therefore, the multi-VNS algorithm in this paper runs the $N$ VNS algorithms in order from the 1st VNS to the $N$ th VNS, sequentially. The procedure of the generic multi-VNS algorithm used in this paper is given in Algorithm 3.

Algorithm 3. It is a procedure of the generic multi-VNS algorithm.

Step 1. Assign the input parameter values as follows.

Step 1.1. Specify the specific 1st VNS, 2nd VNS, ..., Nth VNS algorithms for the multi-VNS algorithm differently in their combinations of the operators $A, B, C$, and $D$ and the VNS types. For each VNS algorithm, each of $A, B, C$, and $D$ can be either the swap operator or the insert operator, while the VNS type can be either a forward VNS algorithm or a reverse VNS algorithm.

Step 1.2. Assign the stopping criterion for each of the 1st VNS, 2nd VNS, ..., Nth VNS algorithms.

Step 1.3. Assign the stopping criterion for the multi-VNS algorithm.

Step 2. Randomly generate $P_{0}$ as an identical initial operation-based permutation for all $N$ VNS algorithms. Let the multi-VNS algorithm's iteration $d=1$.

Step 3. Run the $d$ th VNS algorithm using the permutation $P_{0}$ generated in Step 2 as its initial current best operation-based permutation. 
Step 4. After the $d$ th VNS algorithm is stopped, let $S_{0}^{d}$ be equal to $S_{0}$ of the $d$ th VNS algorithm if the $d$ th VNS algorithm is a forward VNS algorithm; however, let $S_{0}^{d}$ be equal to $S_{R 0}$ of the $d$ th VNS algorithm if the $d$ th VNS algorithm is a reverse VNS algorithm. (Remember that $S_{0}$ is the final solution for the forward VNS algorithm, while $S_{R 0}$ is the final solution for the reverse VNS algorithm.)

Step 5. Update the best found solution of the multi-VNS algorithm or $S^{*}$ using Steps 5.1 and 5.2.

Step 5.1. If $d=1$, let $S^{*}$ equal $S_{0}^{d}$ and let the makespan of $S^{*}$ equal the makespan of $S_{0}^{d}$.

Step 5.2. If $d \geq 2$ and the makespan of $S_{0}^{d}$ is less than the makespan of $S^{*}$, update $S^{*}$ to equal $S_{0}^{d}$ and also update the makespan of $S^{*}$ to equal the makespan of $S_{0}^{d}$.

Step 6. If the stopping criterion of the multi-VNS algorithm is met, then stop and let the final result of the multi-VNS algorithm equal $S^{*}$; otherwise, increase the value of $d$ by 1 and repeat from Step 3.

Algorithm 3 is the generic multi-VNS algorithm where the user must specify the 1st VNS algorithm to the Nth VNS algorithm in their $A, B, C$, and $D$ operators, VNS types, and stopping conditions. These $N$ VNS algorithms of the multi-VNS algorithm in Algorithm 3 must be selected very carefully because more VNS algorithms used in the multiVNS algorithm may consume more resources, especially the computational time, without any guarantees to find a better solution. Moreover, the uses of the same set of the $N$ specific VNS algorithms in different orders may consume different computational times. This is because the stopping criterion of the multi-VNS algorithm is specified to stop when either the $N$ th iteration is reached $(d=N)$ or the optimal solution given by the published literature is found; thus, if the optimal solution is found by the $d$ th VNS algorithm, the multi-VNS algorithm will stop at the $d$ th VNS algorithm and will not continue running the $N-d$ remaining VNS algorithms. Hence, to make the multi-VNS algorithm perform most efficiently in computational time, the order of the $N$ VNS algorithms must be selected carefully as well. In this paper, the method of selecting the proper value of $N$ and also selecting the specific 1st VNS to Nth VNS algorithms for the multi-VNS algorithm is given in Algorithm 4. Remember that, for each instance, the solution value deviation of each VNS algorithm is equal to $100 \% \times$ (the VNS algorithm's final solution value - the optimal solution value)/the optimal solution value.

Algorithm 4. It is a method of selecting the value of $N$ and selecting the specific 1st to Nth VNS algorithms for the multiVNS algorithm.

Step 1. Let $d=1$. Run all on-hand VNS algorithms once on all given instances and then receive the final solutions of all these on-hand VNS algorithms. Then, compute the following based on the final solutions received.
TABLE 3: The 1st VNS to $d$ th VNS algorithms and $A B S V D_{d}$ value in each iteration of Algorithm 4 based on results from Table 1.

\begin{tabular}{lcc}
\hline$d$ & The 1st VNS to $d$ th VNS & $A B S V D_{d}$ \\
\hline All & All 32 VNS algorithms & $0.020 \%$ \\
1 & FSSII & $0.057 \%$ \\
2 & FSSII, FISIS & $0.037 \%$ \\
3 & FSSII, FISIS, FSSSI & $0.027 \%$ \\
4 & FSSII, FISIS, FSSSI, RSSIS & $0.020 \%$ \\
\hline
\end{tabular}

Step 1.1. For each on-hand VNS algorithm, compute the solution value deviation of every given instance and then compute the average of the solution value deviations of all given instances. Then, list all on-hand VNS algorithms in ascending order of their average solution value deviations (from left to right).

Step 1.2. For every given instance, find $B S V D_{\text {All }}$, that is, the best (lowest) solution value deviation found by all onhand VNS algorithms. Then, compute $A B S V D_{\text {All }}$, that is, the average of the $B S V D_{\text {All }}$ values of all given instances.

Step 2. Assign the current leftmost VNS algorithm among all as-yet-unassigned on-hand VNS algorithms on the list given in Step 1.1 as the $d$ th VNS algorithm.

Step 3. For every given instance, find $B S V D_{d}$, that is, the best (lowest) solution value deviation found by all the alreadyspecified 1st VNS to $d$ th VNS algorithms. Then, compute $A B S V D_{d}$, that is, the average of the $B S V D_{d}$ values of all given instances.

Step 4. If $A B S V D_{d}$ is equal to $A B S V D_{\text {All }}$, then stop and let $N$ equal $d$; this means the specific 1 st to $N$ th VNS algorithms are completely selected. On the other hand, if $A B S V D_{d}$ is greater than $A B S V D_{\text {All }}$, then increase $d$ by 1 and repeat from Step 2 .

As shown in Section 3.2, this paper proposes 16 FVNS and 16 RVNS algorithms, so the number of all on-hand VNS algorithms is thus 32 . Based on the results from Table 1 , the list of all 32 VNS algorithms in ascending order of their average solution value deviations on all 43 instances is FSSII, FISIS, FSSSI, RSSIS, RSISI, RISSI, FIIII, RIISI, RSSSI, FSIII, RIISS, FISSS, RSSSS, RISIS, RISII, RIIII, FSIIS, RIIIS, FIIIS, RSSII, RSIII, FIISI, FSSIS, RSIIS, FSSSS, FSISI, FISSI, RISSS, FIISS, FISII, RSISS, and FSISS. According to the results in Table 1, Algorithm 4 first computes $A B S V D_{\text {All }}=0.020 \%$. After that, Algorithm 4 assigns FSSII as the 1st VNS algorithm with $A B S V D_{1}=0.057 \%$, FISIS as the 2nd VNS algorithm with $A B S V D_{2}=0.037 \%$, FSSSI as the 3rd VNS algorithm with $A B S V D_{3}=0.027 \%$, and RSSIS as the 4th VNS algorithm with $A B S V D_{4}=0.020 \%$, respectively. Since $A B S V D_{4}$ equals $A B S V D_{\text {All }}$, Algorithm 4 stops here and thus $N=4$. This means, based on Table 1 results, Algorithm 4 suggests that the multi-VNS algorithm should use FSSII, FISIS, FSSSI, and RSSIS as the 1st to 4 th VNS algorithms, respectively. Table 3 summarizes the specified 1st VNS algorithm to the $d$ th VNS 
algorithm and the $A B S V D_{d}$ value given in each iteration of Algorithm 4 based on the results from Table 1.

\section{Performance Evaluation for Multi-VNS}

This section will provide a specific multi-VNS algorithm, which is Algorithm 3 using the $N$ specific VNS algorithms and the other preassigned input parameter values. Later, an experiment will be conducted in order to evaluate the performance of the multi-VNS algorithm on the JSP instances. The specific VNS algorithms and input parameter values for Algorithm 3 along with the experimental conditions are given below.

(1) The multi-VNS algorithm uses the four specified VNS algorithms $(N=4)$, that is, FSSII, FISIS, FSSSI, and RSSIS as the 1st VNS, 2nd VNS, 3rd VNS, and 4th VNS algorithms, respectively. (Note that these VNS algorithms are selected by Algorithm 4 based on Table 1 results.)

(2) The stopping criterion of each specified VNS algorithm (i.e., FSSII, FISIS, FSSSI, and RSSIS) used in the multi-VNS algorithm is to stop when either the optimal solution value (i.e., the optimal makespan) given by the published literature is found or the 1,000 th iteration is reached $(t=1,000)$.

(3) The stopping criterion of the multi-VNS algorithm is to stop when either the optimal solution value (i.e., the optimal makespan) given by the published literature is found or the multi-VNS algorithm's $N$ th iteration is reached.

(4) On each single run of the multi-VNS algorithm, the multi-VNS algorithm runs the specific 1st VNS, 2nd VNS, 3rd VNS, and 4th VNS algorithms with the same random seed number and also the same initial operation-based permutation.

(5) The multi-VNS algorithm is coded in C\# and executed on an Intel(R) Core(TM) i5 CPU processor M580 $2.67 \mathrm{GHz}$.

(6) The multi-VNS algorithm in this paper will be repeated for five runs with different random seed numbers and also different initial operation-based permutations. The multi-VNS algorithm in the 1st run uses the same random seed number and the same initial operation-based permutation as the VNS algorithms in the experiment in Table 1.

(7) The performance of the multi-VNS algorithm will be tested on the 43 benchmark JSP instances including $\mathrm{ft} 06, \mathrm{ft} 10$, and $\mathrm{ft} 20$ from [34] and la01 to la40 from [35].

Table 4 shows the final solution values from the five runs of the multi-VNS algorithm with the above-given settings. The column Best in Table 4 provides the best among the final solution values of the five runs for every instance. The column Avg provides the average of the final solution values of the five runs. The column Avg CPU time (sec.) provides the

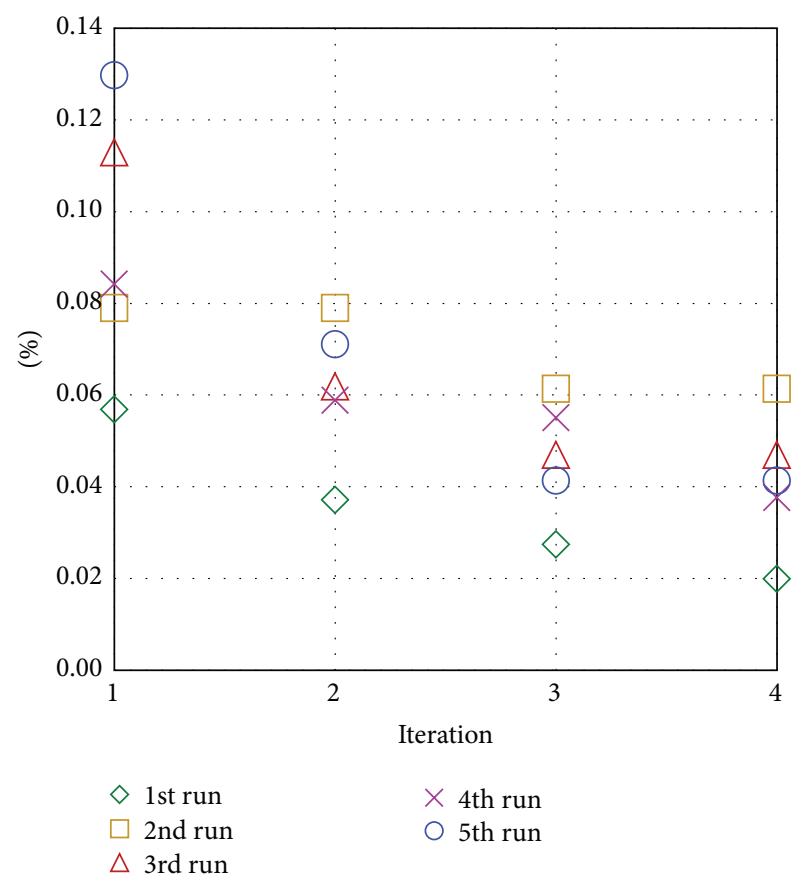

FIGURE 3: Average-solution-value-deviation-over-iteration plots for five runs of the multi-VNS algorithm.

average computational time in second over the five runs of each instance.

Figure 3 shows the average solution value deviations of 43 instances over the multi-VNS algorithm's iterations ( $d$ values) of each run. The average solution value deviation in Figure 3 is, on average, reduced in high rate from the 1st iteration to the 3rd iteration and then reduced in lower rate from the 3 rd iteration to the 4 th iteration. Figure 3 also shows that the multi-VNS algorithm improves the average solution value deviation of its 1 st VNS (i.e., FSSII here) for $0.05 \%$ on average. The rate of improvement of $0.05 \%$ may seem to be low; however, if considering only the instances where FSSII cannot find the optimal solutions in Table 1 experiment (i.e., la24, la29, la38, and la40), the rate of improvement will be $0.21 \%$ on average.

Later, the performance of the multi-VNS algorithm is then compared to the performances of the five highperforming metaheuristic algorithms in published literature, that is, the GA with the extended Akers graphical method [8], the hybrid GA [9], the two-level PSO [14], the VNS algorithm [17], the memetic algorithm [23], and also FSSII, as the best-performing VNS algorithm in Table 1. In this section, the performances of these algorithms are compared only in their best found solution values. The computational times of these algorithms will not be compared here because of the differences in their stopping criteria, programming languages, and CPU processor specifications. The best found solution values of the algorithms in $[8,9,14,17]$ are taken from their own articles, the best found solution values of the multi-VNS algorithm are taken from the column Best in Table 4, and the best found solution values of FSSII are taken from the results over 5 runs in an additional experiment here. 
TABLE 4: Final solution value by each run of the multi-VNS algorithm.

\begin{tabular}{|c|c|c|c|c|c|c|c|c|}
\hline Instance & 1st run & 2nd run & 3rd run & 4 th run & 5 th run & Best & Avg & Avg CPU time (sec.) \\
\hline $\mathrm{ft} 06$ & 55 & 55 & 55 & 55 & 55 & 55 & 55 & 0.1 \\
\hline $\mathrm{ft} 10$ & 930 & 930 & 930 & 930 & 930 & 930 & 930 & 546 \\
\hline $\mathrm{ft} 20$ & 1165 & 1165 & 1165 & 1165 & 1165 & 1165 & 1165 & 382 \\
\hline la01 & 666 & 666 & 666 & 666 & 666 & 666 & 666 & 0.3 \\
\hline la02 & 655 & 655 & 655 & 655 & 655 & 655 & 655 & 2 \\
\hline la03 & 597 & 597 & 597 & 597 & 597 & 597 & 597 & 37 \\
\hline la04 & 590 & 590 & 590 & 590 & 590 & 590 & 590 & 0.5 \\
\hline la05 & 593 & 593 & 593 & 593 & 593 & 593 & 593 & 0.2 \\
\hline la06 & 926 & 926 & 926 & 926 & 926 & 926 & 926 & 1 \\
\hline la07 & 890 & 890 & 890 & 890 & 890 & 890 & 890 & 1 \\
\hline la08 & 863 & 863 & 863 & 863 & 863 & 863 & 863 & 1 \\
\hline la09 & 951 & 951 & 951 & 951 & 951 & 951 & 951 & 1 \\
\hline la10 & 958 & 958 & 958 & 958 & 958 & 958 & 958 & 1 \\
\hline lall & 1222 & 1222 & 1222 & 1222 & 1222 & 1222 & 1222 & 2 \\
\hline la12 & 1039 & 1039 & 1039 & 1039 & 1039 & 1039 & 1039 & 2 \\
\hline la13 & 1150 & 1150 & 1150 & 1150 & 1150 & 1150 & 1150 & 2 \\
\hline la14 & 1292 & 1292 & 1292 & 1292 & 1292 & 1292 & 1292 & 3 \\
\hline la15 & 1207 & 1207 & 1207 & 1207 & 1207 & 1207 & 1207 & 2 \\
\hline la16 & 945 & 945 & 945 & 945 & 945 & 945 & 945 & 260 \\
\hline la17 & 784 & 784 & 784 & 784 & 784 & 784 & 784 & 42 \\
\hline la18 & 848 & 848 & 848 & 848 & 848 & 848 & 848 & 16 \\
\hline la19 & 842 & 842 & 842 & 842 & 842 & 842 & 842 & 30 \\
\hline la 20 & 902 & 902 & 902 & 902 & 902 & 902 & 902 & 522 \\
\hline la21 & 1046 & 1046 & 1046 & 1047 & 1047 & 1046 & 1046.4 & 8526 \\
\hline la22 & 927 & 927 & 927 & 927 & 927 & 927 & 927 & 995 \\
\hline la23 & 1032 & 1032 & 1032 & 1032 & 1032 & 1032 & 1032 & 11 \\
\hline la24 & 935 & 938 & 938 & 938 & 938 & 935 & 937.4 & 14258 \\
\hline la25 & 977 & 977 & 977 & 977 & 977 & 977 & 977 & 4187 \\
\hline la26 & 1218 & 1218 & 1218 & 1218 & 1218 & 1218 & 1218 & 26 \\
\hline la27 & 1235 & 1235 & 1237 & 1236 & 1238 & 1235 & 1236.2 & 14368 \\
\hline la28 & 1216 & 1216 & 1216 & 1216 & 1216 & 1216 & 1216 & 497 \\
\hline la29 & 1160 & 1172 & 1163 & 1163 & 1163 & 1160 & 1164.2 & 42644 \\
\hline la30 & 1355 & 1355 & 1355 & 1355 & 1355 & 1355 & 1355 & 12 \\
\hline la31 & 1784 & 1784 & 1784 & 1784 & 1784 & 1784 & 1784 & 61 \\
\hline la32 & 1850 & 1850 & 1850 & 1850 & 1850 & 1850 & 1850 & 56 \\
\hline la33 & 1719 & 1719 & 1719 & 1719 & 1719 & 1719 & 1719 & 59 \\
\hline la34 & 1721 & 1721 & 1721 & 1721 & 1721 & 1721 & 1721 & 55 \\
\hline la35 & 1888 & 1888 & 1888 & 1888 & 1888 & 1888 & 1888 & 62 \\
\hline la36 & 1268 & 1268 & 1268 & 1268 & 1268 & 1268 & 1268 & 4843 \\
\hline la37 & 1397 & 1397 & 1397 & 1397 & 1397 & 1397 & 1397 & 3192 \\
\hline la38 & 1196 & 1201 & 1201 & 1196 & 1196 & 1196 & 1198 & 40265 \\
\hline la39 & 1233 & 1233 & 1233 & 1233 & 1233 & 1233 & 1233 & 5941 \\
\hline la 40 & 1224 & 1224 & 1224 & 1224 & 1224 & 1224 & 1224 & 48849 \\
\hline
\end{tabular}

The stopping criterion of FSSII is to stop when either the optimal solution given in the published literature is found or the 1,000th iteration is met. VNS in [17] is the FSISI algorithm which uses the stopping criterion that is to stop when either the optimal solution given in the published literature is found, the 1,000th iteration is reached, or no solution improvements within 250 consecutive iterations happen. The comparison results are then given in Table 5. For each instance, Table 5 provides the best found solution value of each algorithm compared to the optimal solution value given in $[7,8,24]$. 
TABLE 5: Comparison of best found solution values of algorithms.

\begin{tabular}{|c|c|c|c|c|c|c|c|c|}
\hline Instance & Optimal solution value & Multi-VNS & FSSII & GA [8] & GA [9] & PSO [14] & VNS [17] & MA [23] \\
\hline $\mathrm{ft} 06$ & 55 & 55 & 55 & 55 & 55 & 55 & $\mathrm{n} / \mathrm{a}$ & 55 \\
\hline $\mathrm{ft} 10$ & 930 & 930 & 930 & 930 & 930 & 930 & $\mathrm{n} / \mathrm{a}$ & 930 \\
\hline $\mathrm{ft} 20$ & 1165 & 1165 & 1165 & 1165 & 1165 & 1165 & $\mathrm{n} / \mathrm{a}$ & 1165 \\
\hline la01 & 666 & 666 & 666 & 666 & 666 & 666 & 666 & 666 \\
\hline la02 & 655 & 655 & 655 & 655 & 655 & 655 & 655 & 655 \\
\hline la03 & 597 & 597 & 597 & 597 & 597 & 597 & 597 & 597 \\
\hline la04 & 590 & 590 & 590 & 590 & 590 & 590 & 590 & 590 \\
\hline la05 & 593 & 593 & 593 & 593 & 593 & 593 & 593 & 593 \\
\hline la06 & 926 & 926 & 926 & 926 & 926 & 926 & 926 & 926 \\
\hline la07 & 890 & 890 & 890 & 890 & 890 & 890 & 890 & 890 \\
\hline la08 & 863 & 863 & 863 & 863 & 863 & 863 & 863 & 863 \\
\hline la09 & 951 & 951 & 951 & 951 & 951 & 951 & 951 & 951 \\
\hline la10 & 958 & 958 & 958 & 958 & 958 & 958 & 958 & 958 \\
\hline lall & 1222 & 1222 & 1222 & 1222 & 1222 & 1222 & 1222 & 1222 \\
\hline la12 & 1039 & 1039 & 1039 & 1039 & 1039 & 1039 & 1039 & 1039 \\
\hline la13 & 1150 & 1150 & 1150 & 1150 & 1150 & 1150 & 1150 & 1150 \\
\hline la14 & 1292 & 1292 & 1292 & 1292 & 1292 & 1292 & 1292 & 1292 \\
\hline la15 & 1207 & 1207 & 1207 & 1207 & 1207 & 1207 & 1207 & 1207 \\
\hline la16 & 945 & 945 & 945 & 945 & 945 & 945 & 945 & 945 \\
\hline lal7 & 784 & 784 & 784 & 784 & 784 & 784 & 784 & 784 \\
\hline la18 & 848 & 848 & 848 & 848 & 848 & 848 & 848 & 848 \\
\hline la19 & 842 & 842 & 842 & 842 & 842 & 842 & 842 & 842 \\
\hline la20 & 902 & 902 & 902 & 902 & 907 & 907 & 902 & 902 \\
\hline la21 & 1046 & 1046 & 1046 & 1046 & 1046 & 1046 & 1047 & 1055 \\
\hline la22 & 927 & 927 & 927 & 927 & 927 & 935 & 927 & 927 \\
\hline la23 & 1032 & 1032 & 1032 & 1032 & 1032 & 1032 & 1032 & 1032 \\
\hline la24 & 935 & 935 & 938 & 935 & 953 & 944 & 937 & 940 \\
\hline la25 & 977 & 977 & 977 & 977 & 986 & 984 & 977 & 984 \\
\hline la26 & 1218 & 1218 & 1218 & 1218 & 1218 & 1218 & 1218 & 1218 \\
\hline la27 & 1235 & 1235 & 1235 & 1235 & 1256 & 1258 & 1235 & 1261 \\
\hline la28 & 1216 & 1216 & 1216 & 1216 & 1232 & 1218 & 1216 & 1216 \\
\hline la29 & 1152 & 1160 & 1164 & 1153 & 1196 & 1184 & 1163 & 1190 \\
\hline la30 & 1355 & 1355 & 1355 & 1355 & 1355 & 1355 & 1355 & 1355 \\
\hline la31 & 1784 & 1784 & 1784 & 1784 & 1784 & 1784 & 1784 & 1784 \\
\hline la32 & 1850 & 1850 & 1850 & 1850 & 1850 & 1850 & 1850 & 1850 \\
\hline la33 & 1719 & 1719 & 1719 & 1719 & 1719 & 1719 & 1719 & 1719 \\
\hline la34 & 1721 & 1721 & 1721 & 1721 & 1721 & 1721 & 1721 & 1721 \\
\hline la35 & 1888 & 1888 & 1888 & 1888 & 1888 & 1888 & 1888 & 1888 \\
\hline la36 & 1268 & 1268 & 1268 & 1268 & 1279 & 1278 & 1268 & 1281 \\
\hline la37 & 1397 & 1397 & 1397 & 1397 & 1408 & 1410 & 1397 & 1431 \\
\hline la38 & 1196 & 1196 & 1201 & 1196 & 1219 & 1221 & 1201 & 1216 \\
\hline la39 & 1233 & 1233 & 1233 & 1233 & 1246 & 1251 & 1233 & 1241 \\
\hline la 40 & 1222 & 1224 & 1224 & 1222 & 1241 & 1229 & 1224 & 1233 \\
\hline Avg deviation & $0.000 \%$ & $0.020 \%$ & $0.045 \%$ & $0.002 \%$ & $0.382 \%$ & $0.320 \%$ & $0.046 \%$ & $0.330 \%$ \\
\hline
\end{tabular}


For each algorithm, the row Avg deviation in Table 5 provides the average of the solution value deviations of all best found solutions.

According to the results in Table 5, GA [8] performs best with the average deviation of $0.002 \%$; it can find the optimal solutions for 42 instances over all 43 instances. The multi-VNS performs as the second-best with the average deviation of $0.020 \%$, and it can find the optimal solutions for 41 instances. The algorithms FSSII and VNS [17] perform equally well as the third and the fourth and are followed by PSO [14], GA [9], and MA [23].

\section{Conclusions}

This paper proposed the 16 forward VNS algorithms and the 16 reverse VNS algorithms for JSP. It has been found that, on many benchmark instances, the VNS algorithms perform unequally even when using the same initial operation-based permutation. Thus, for utilizing each initial operation-based permutation most efficiently, this paper developed the multiVNS algorithm which assigns the same initial operationbased permutation into the selected specific VNS algorithms (i.e., FSSII, FISIS, FSSSI, and RSSIS in this paper), runs these VNS algorithms, and uses the best solution found by all these VNS algorithms as its final result. This paper then compared the multi-VNS algorithm's performance with the performances of the six other high-performing algorithms. The comparison results indicate that the multi-VNS algorithm is the second-best algorithm in terms of solution quality. Over all the 43 benchmark instances used, the multi-VNS algorithm can find the optimal solutions for the 41 instances and the very-near optimal solutions for the two instances. The further work of this research is enhancing the performances of the VNS algorithms in terms of both solution quality and computational time by applying a combination of multiple techniques.

\section{Competing Interests}

The author declares that there are no competing interests.

\section{References}

[1] K. R. Baker and D. Trietsch, Principles of Sequencing and Scheduling, John Wiley \& Sons, Hoboken, NJ, USA, 2009.

[2] M. L. Pinedo, Scheduling: Theory, Algorithms, and Systems, Springer, New York, NY, USA, 4th edition, 2012.

[3] M. Gen and R. Cheng, Genetic Algorithms and Engineering Design, John Wiley \& Sons, New York, NY, USA, 1996.

[4] E. Nowicki and C. Smutnicki, "An advanced tabu search algorithm for the job shop problem," Journal of Scheduling, vol. 8, no. 2, pp. 145-159, 2005.

[5] C. Y. Zhang, P. Li, Z. Guan, and Y. Rao, "A tabu search algorithm with a new neighborhood structure for the job shop scheduling problem," Computers \& Operations Research, vol. 34, no. 11, pp. 3229-3242, 2007.

[6] R. K. Suresh and K. M. Mohanasundaram, "Pareto archived simulated annealing for job shop scheduling with multiple objectives," International Journal of Advanced Manufacturing Technology, vol. 29, no. 1-2, pp. 184-196, 2006.

[7] M. F. Tasgetiren, M. Sevkli, Y.-C. Liang, and M. M. Yenisey, "A particle swarm optimization and differential evolution algorithms for job shop scheduling problem," International Journal of Operations Research, vol. 3, no. 2, pp. 120-135, 2006.

[8] J. F. Gonçalves and M. G. C. Resende, "An extended Akers graphical method with a biased random-key genetic algorithm for job-shop scheduling," International Transactions in Operational Research, vol. 21, no. 2, pp. 215-246, 2014.

[9] J. F. Gonçalves, J. J. Mendes, and M. G. Resende, "A hybrid genetic algorithm for the job shop scheduling problem," European Journal of Operational Research, vol. 167, no. 1, pp. 77-95, 2005.

[10] M. Watanabe, K. Ida, and M. Gen, "A genetic algorithm with modified crossover operator and search area adaptation for the job-shop scheduling problem," Computers and Industrial Engineering, vol. 48, no. 4, pp. 743-752, 2005.

[11] M. F. N. Maghfiroh, A. Darnawan, and V. F. Yu, "Genetic algorithm for job shop scheduling problem: a case study," International Journal of Innovation, Management and Technology, vol. 4, no. 1, pp. 137-140, 2013.

[12] N. H. Moin, O. C. Sin, and M. Omar, "Hybrid genetic algorithm with multiparents crossover for job shop scheduling problems," Mathematical Problems in Engineering, vol. 2015, Article ID 210680, 12 pages, 2015.

[13] M. Gen, Y. Tsujimura, and E. Kubota, "Solving job-shop scheduling problem using genetic algorithms," in Proceedings of the 16th International Conference on Computers and Industrial Engineering, pp. 576-579, Ashikaga, Japan, 1994.

[14] P. Pongchairerks and V. Kachitvichyanukul, "A two-level particle swarm optimization algorithm on job-shop scheduling problems," International Journal of Operational Research, vol. 4, no. 4, pp. 390-411, 2009.

[15] P. Pongchairerks, "Particle swarm optimization algorithm applied to scheduling problems," ScienceAsia, vol. 35, no. 1, pp. 89-94, 2009.

[16] P. Pongchairerks, "A self-tuning PSO for job-shop scheduling problems," International Journal of Operational Research, vol. 19, no. 1, pp. 96-113, 2014.

[17] P. Pongchairerks and V. Kachitvichyanukul, "A comparison between algorithms VNS with PSO and VNS without PSO for job-shop scheduling problems," International Journal of Computational Science, vol. 1, no. 2, pp. 179-191, 2007.

[18] M. Sevkli and M. E. Aydin, "Variable neighbourhood search for job shop scheduling problems," Journal of Software, vol. 1, no. 2, pp. 34-39, 2006.

[19] P. Pongchairerks, "Variable neighbourhood search algorithms applied to job-shop scheduling problems," International Journal of Mathematics in Operational Research, vol. 6, no. 6, pp. 752774, 2014.

[20] L.-L. Liu, R.-S. Hu, X.-P. Hu, G.-P. Zhao, and S. Wang, "A hybrid PSO-GA algorithm for job shop scheduling in machine tool production," International Journal of Production Research, vol. 53, no. 19, pp. 5755-5781, 2015.

[21] J.-Q. Li, S.-X. Xie, Q.-K. Pan, and S. Wang, "A hybrid artificial bee colony algorithm for flexible job shop scheduling problems," International Journal of Computers, Communications and Control, vol. 6, no. 2, pp. 286-296, 2011.

[22] A. Udomsakdigool and V. Kachitvichyanukul, "Two-way scheduling approach in ant algorithm for solving job shop 
problem," Industrial Engineering and Management Systems, vol. 5, no. 2, pp. 68-75, 2007.

[23] L. Gao, G. Zhang, L. Zhang, and X. Li, "An efficient memetic algorithm for solving the job shop scheduling problem," Computers and Industrial Engineering, vol. 60, no. 4, pp. 699-705, 2011.

[24] C. Zhang, P. Li, Y. Rao, and S. Li, "A new hybrid GA/SA algorithm for the job shop scheduling problem," in Proceedings of the 5th European Conference on Evolutionary Computation in Combinatorial Optimization (EvoCOP '05), pp. 246-259, Lausanne, Switzerland, April 2005.

[25] N. Mladenović and P. Hansen, "Variable neighborhood search," Computers and Operations Research, vol. 24, no. 11, pp. 10971100, 1997.

[26] P. Hansen and N. Mladenović, "Variable neighborhood search: principles and applications," European Journal of Operational Research, vol. 130, no. 3, pp. 449-467, 2001.

[27] J. A. Moreno-Pérez, P. Hansen, and N. Mladenović, "Parallel variable neighborhood searchs," Tech. Rep., Group of Intelligent Computing, Universidad de La Laguna, La Laguna, Spain, 2004.

[28] M. M. Gargari and M. S. F. Niasar, "A dynamic discrete berth allocation problem for container terminals," in Proceedings of the Conference on Maritime-Port Technology, Trondheim, Norway, 2014.

[29] I. Piriyaniti and P. Pongchairerks, "Variable neighbourhood search algorithms for asymmetric travelling salesman problems," International Journal of Operational Research, vol. 18, no. 2, pp. 157-170, 2013.

[30] T. Davidovic, T. G. Crainic, and T. Davidović, "Parallelization strategies for variable neighborhood search," Tech. Rep., CIRRELT, Université de Montréal, Montreal, Canada, 2013.

[31] T. Yamada and R. Nakano, "Fusion of crossover and local search," in Proceedings of the IEEE International Conference on Industrial Technology, pp. 426-430, Shanghai, China, December 1994.

[32] J. Alcaraz and C. Maroto, "A robust genetic algorithm for resource allocation in project scheduling," Annals of Operations Research, vol. 102, no. 1-4, pp. 83-109, 2001.

[33] E. J. Anderson, C. A. Glass, and C. N. Potts, "Machine scheduling," in Local Search in Combinatorial Optimization, E. Aarts and J. K. Lenstra, Eds., pp. 361-414, Princeton University Press, Princeton, NJ, USA, 2003.

[34] H. Fisher and G. L. Thompson, "Probabilistic learning combinations of local job-shop scheduling rules," in Industrial Scheduling, J. F. Muth and G. L. Thompson, Eds., pp. 225-251, Prentice-Hall, Englewood Cliffs, NJ, USA, 1963.

[35] S. Lawrence, Resource Constrained Project Scheduling: An Experimental Investigation of Heuristic Scheduling Techniques, Graduate School of Industrial Administration, Carnegie-Mellon University, Pittsburgh, Pa, USA, 1984. 


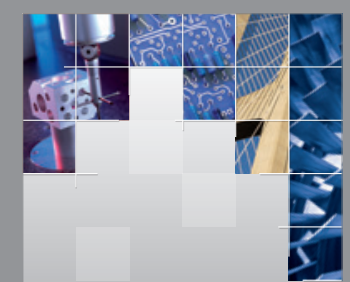

\section{Enfincering}
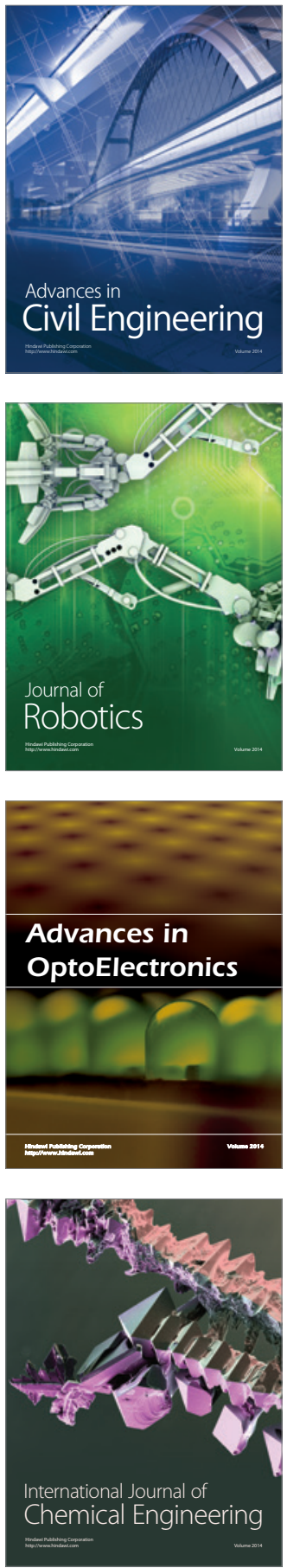

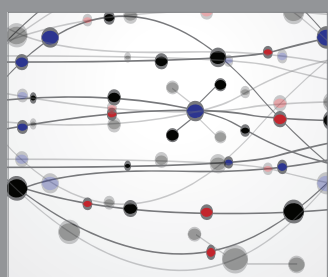

The Scientific World Journal

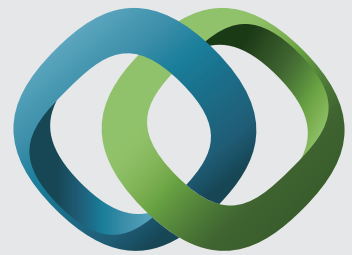

\section{Hindawi}

Submit your manuscripts at

http://www.hindawi.com
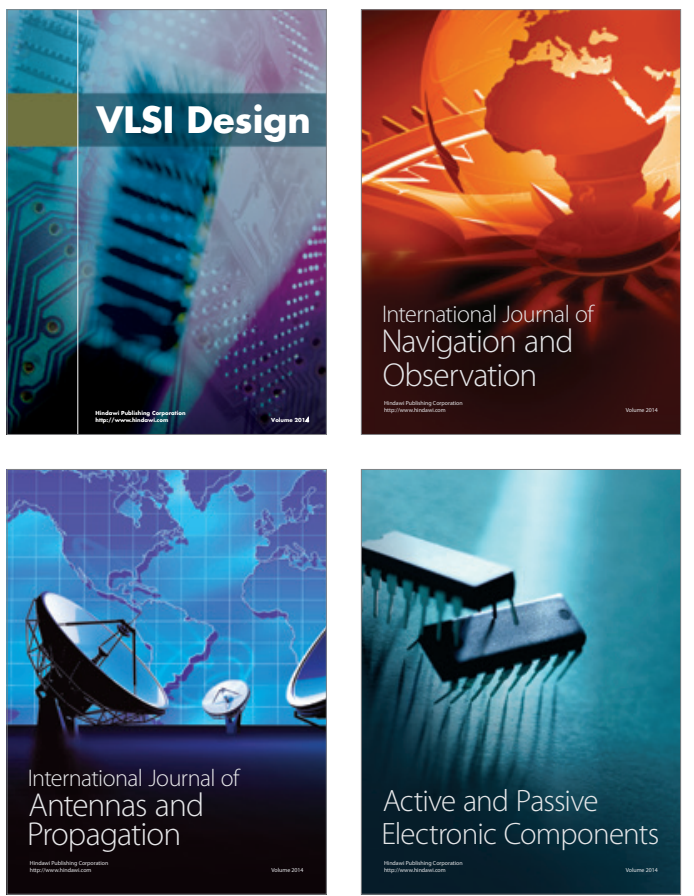
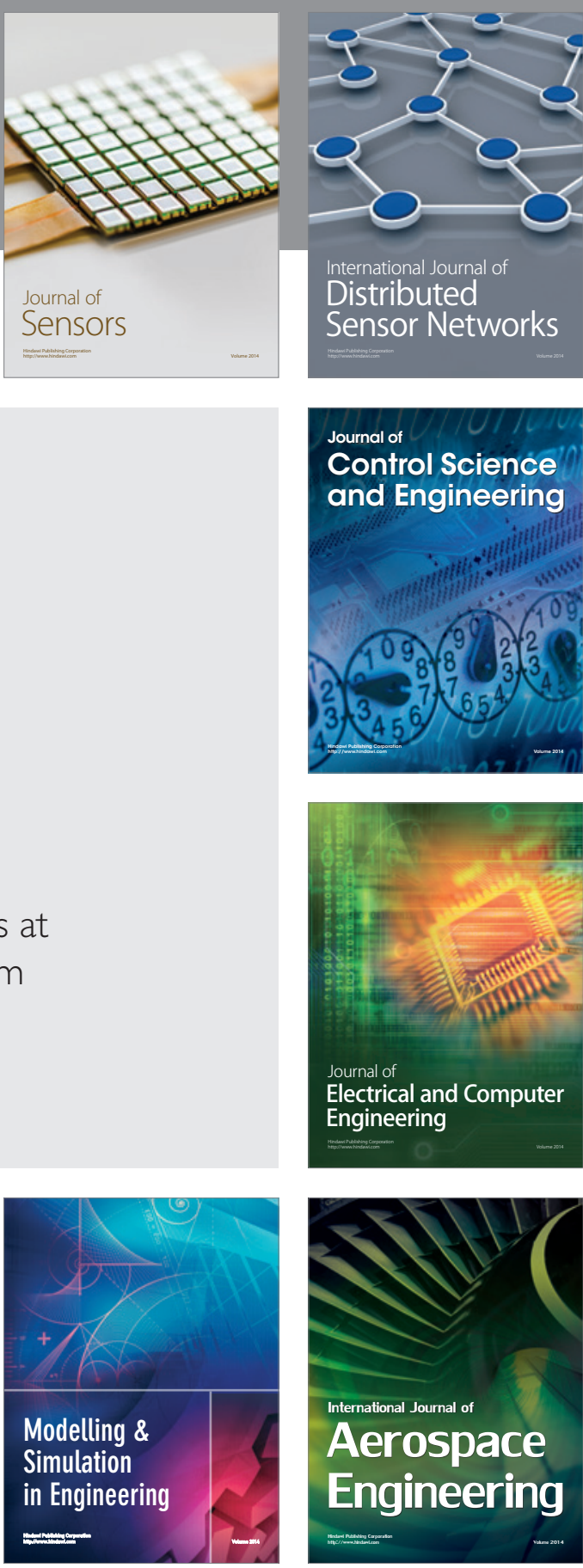

International Journal of

Distributed

Sensor Networks

Journal of

Control Science

and Engineering
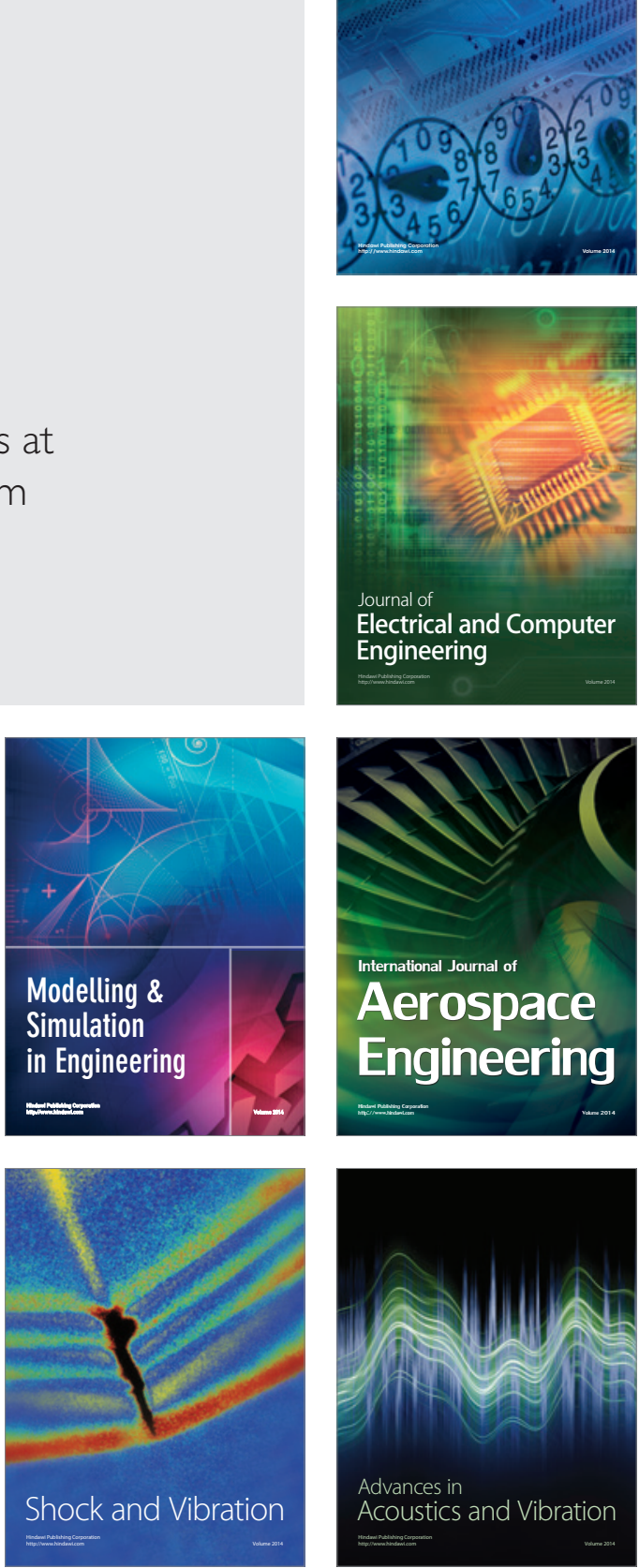